\title{
Measurement of Stresses in Fixed-Bridge Restorations Using a Brittle Coating Technique
}

\author{
R. G. CRAIG and F. A. PEYTON \\ School of Dentistry, University of Michigan, Ann Arbor, Michigan
}

Three principal methods used to study stresses in materials are photoelastic stress analysis, brittle lacquer-coating techniques, and electronic strain gauges. A number of studies have been conducted using models and the photoelastic method ${ }^{1-8}$ to examine stress patterns in one- and two-surface inlays, complete dentures, teeth and supporting bone, and rests used in partial dentures. Strain gauges ${ }^{4,9-13}$ have been used in the evaluations of stresses of mastication, but brittle lacquer coatings have not been used to any extent in the measurement of stresses in dental restorations. ${ }^{4}$ Brittle lacquers have been used in the study of stresses in the human mandible and other bones under static and dynamic loading. ${ }^{14-17}$ The brittle lacquer coatings are particularly useful in detecting and measuring strains at the surface of the structure as well as indicating the direction and sequence of the tensile strains. Although the method is not as accurate as strain gauges, it is an excellent means of obtaining an over-all picture of the pattern of the stress distribution. ${ }^{18}$ The information obtained using brittle lacquers may be used, therefore, as a basis for more detailed studies with strain gauges. It was the purpose of this investigation to study the general stress patterns in typical fixedbridge restorations using various static loading conditions and a brittle lacquer-coating technique.

\section{Materials and Methods}

Three bridges were selected as typical restorations used in crown and bridge prosthesis: (1) a six-unit maxillary anterior

Presented at the $42 \mathrm{~d}$ General Meeting of the International Association for Dental Research, Los Angeles, California, March, 1964.

This investigation was supported in whole by Public Health Service Research Grant DE-01817 from the National Institute for Dental Research, National Institutes of Health, Bethesda, Md.

Received for publication May 26, 1964. bridge was prepared using pin-ledge preparations* on the cuspid abutments; (2) a six-unit mandibular bridge was constructed with $\frac{3}{4}$-crown preparations on the cuspid abutments; and (3) a four-unit right maxillary bridge was prepared using a full-crown preparation on the second molar and a $\frac{3}{4}$ crown on the first bicuspid.

The three appropriate models of the upper and lower arch were prepared in acrylic plastic using a rubber mold. $\dagger$ The appropriate teeth were blocked out by filling the space with wax prior to preparation of the plastic model. The Ivorine were placed in the mold and properly lubricated to assure their removal after preparing the model. The preparations were cut in the Ivorine teeth, and the preparations were formed in wax and cast in gold. \& The pontics for each bridge then were formed in wax and cast as a single unit. The pontics and the two abutments were soldered together with a 650 -fineness solder. $\|$ The bridges were cemented to the prepared teeth with zinc phosphate cement, thus completing the assembly of the restorations. It was found on loading the maxillary anterior bridge that the Ivorine abutment teeth were not sufficiently strong to survive the test procedure. As a result the two maxillary cuspids were cast in brass, the preparations were cut in these teeth, and the bridge constructed as previously described. The labial and lingual views of the six-unit maxillary anterior bridge are shown in Figure 1, and those of the six-unit mandibular bridge are pictured in Figure 2. The

\footnotetext{
* Three tapered pins, $3 \mathrm{~mm}$. long, were used which were cut with a $\$ 700$ fissure bur; 2 incisal pins and one singulum pin were used.

$\uparrow$ Columbia Dentoform mold was used to prepare the plastic model.

$\ddagger$ Columbia Dentoform Corp., New York, N.Y.

$\$$ Ney-Oro B-2, with no heat treatment other than slow cooling in the investment and heating during soldering.

\| Ney 650 solder.
} 
buccal and lingual views of the four-unit maxillary posterior bridge are shown in Figure 3 .

The bridges were cleaned with carbon disulfide and sprayed with the desired brittle lacquer.* The selection of the lacquer de- pends on the humidity, temperature, and sensitivity, and the method is described by deForest, Ellis, and Stern. ${ }^{18}$ The lacquer is sprayed so that a uniform coating of $0.005-$

* Stresscoat, for typical weather conditions lacquer ST-1206 was satisfactory. Magnaflux Corporation, Chicago, III.
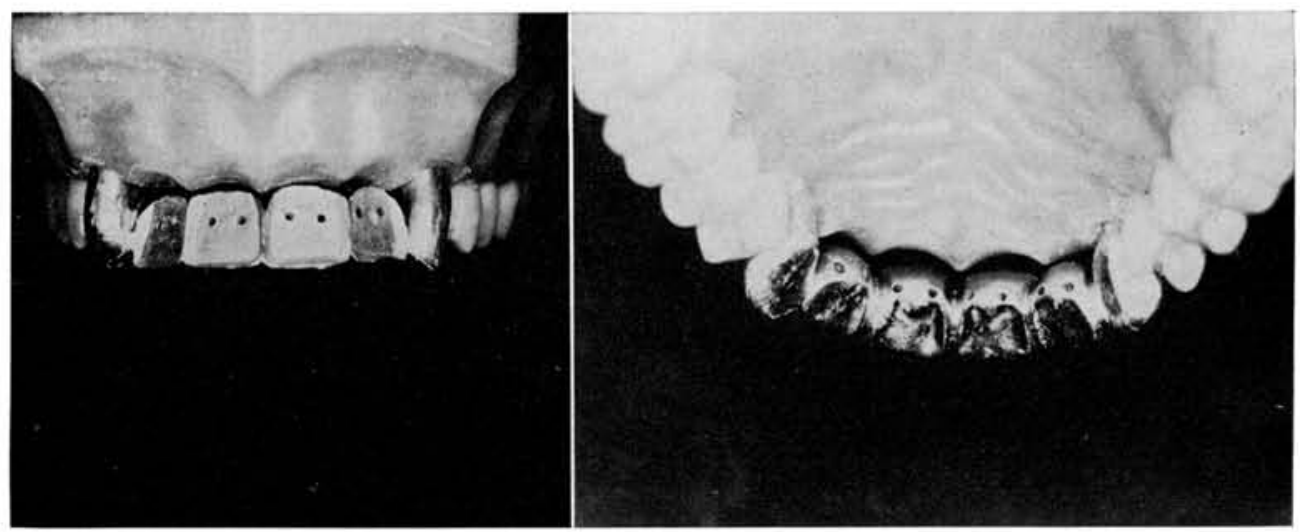

FIG. 1.- Labial and lingual view of the maxillary anterior bridge cemented in position on the model
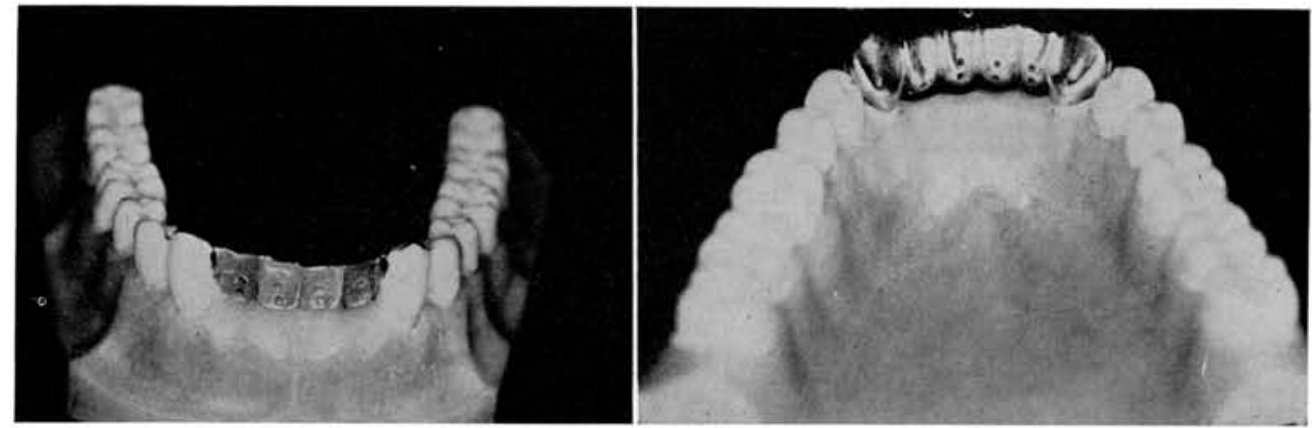

FIG. 2.- Labial and lingual view of the mandibular anterior bridge with three-quarter crowns used as the supporting restorations.
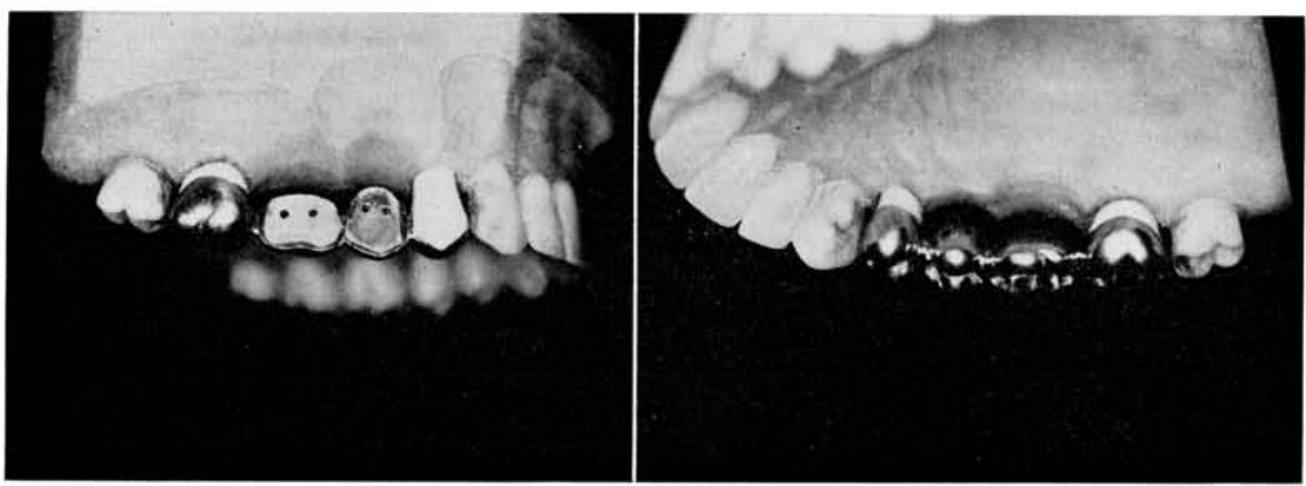

Fig. 3.-Buccal and lingual view of the maxillary posterior bridge cemented in position on the model 
0.006 inches is applied to the bridge. For purposes of calibration, aluminum bars $\frac{1}{4} \times$ $1 \times 12$ inches were sprayed with the same lacquer under identical conditions. An air pressure of 20-psi gauge was adequate, and 3-4 passes of the lacquer spray at a distance of 2.5 inches produced the proper coatings. The bridges, cemented to the abutment teeth, were generally removed from the model during the spraying procedure, and the roots of teeth were protected with masking tape. Immediately after spraying, the masking tape was removed, and the roots of the abutment teeth were placed in the appropriate sockets of the plastic model.

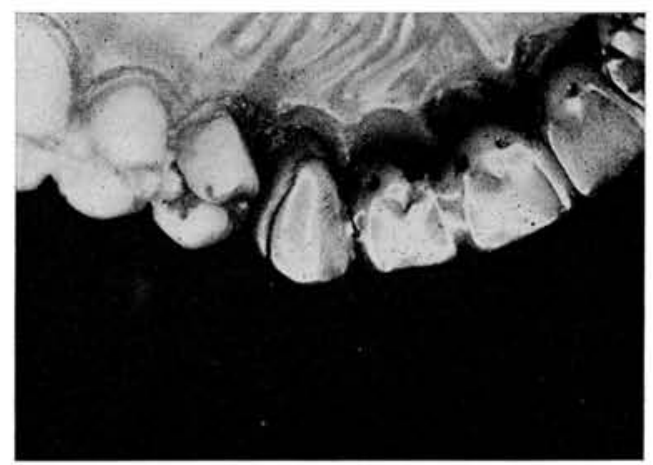

FIG. 4.-Lingual view of the upper anterior bridge has been lacquered, stressed, and etched with a dye to show the cracks on the lingual aspect of the left cuspid.

The models and test bars were allowed to dry in air for $\frac{1}{2}$ hour and then were dried overnight in an air oven at $90^{\circ}-100^{\circ} \mathrm{F}$. The models and test bars were removed from the oven and allowed to stand at room temperature 1 hour before starting the test procedure.

The test bars used for calibrating the lacquer were placed in a device for loading the beam as a cantilever. ${ }^{18}$ One end of the beam is fixed while the other end is deflected in a downward direction by a cam loading mechanism. The calibration bar is placed in the device with a lacquer-coated surface positioned upward so the deflection produces a tensile stress in the lacquer. The calibration of the lacquer is determined by rapidly deflecting the bar and measuring what portion of the length of the lacquer-coating cracked. In most instances the calibration resulted in a value of 800 micro inches/inch for the sensitivity. This means that when- ever the lacquer cracks under a tensile load, the strain at this position has exceeded 800 micro inches/inch. The stress may be calculated by multiplying the sensitivity of the lacquer by the modulus of elasticity of the material which is coated. For example, if a crack is observed in the lacquer coating on one of the gold-alloy bridges during loading, the strain at this position is 800 micro inches/inch and the stress is $0.0008 \times 14 \times$ $10^{6}=11,200 \mathrm{lb} / \mathrm{sq}$ inch. In this instance $14 \times 10^{6}$ is the value of the modulus of elasticity of the alloy.

The models containing the lacquer-coated bridges were placed on the platform of a compression-tension testing machine having a maximum loading capacity of 60 pounds. ${ }^{*}$ The load was applied by a $3-\mathrm{mm}$. strip of tool steel to the incisal edge of the desired central or lateral incisors of the maxillary or mandibular bridges and to the buccal cusp of the posterior bridge. The load was applied to the central fossa of the right first molar by a $\frac{1}{8}$-inch diameter ball bearing. The load was applied in increments of 2 pounds and the lacquer coating was examined for cracks. The load and the position of the cracks were recorded on sketches of the bridges.

The time required to load the bridges to 60 pounds was about 30 minutes. The sensitivity of the lacquer, however, is accurate only if the loading is carried out in 1 second, since the lacquer has a tendency to creep under load. As a result the calibration value of the lacquer must be corrected based on the time elapsed during the test. For example, a typical lacquer having a sensitivity of 800 micro inches/inch when loaded for 30 minutes would have a sensitivity of 820 micro inches/inch. The corresponding stress at the first of the test is $11,200 \mathrm{psi}$, while at the termination of the test the stress in the area of cracks was 11,480 psi.

\section{Results}

In order to simplify the presentation, the loads used have been divided into four ranges: $0-15,16-30,31-45$, and $46-60$ pounds. Cracks in the lacquer in these ranges are indicated in the figures by different types of dotted and dashed lines. An example of the cracks in the lacquer in the preparation of the upper left cuspid is shown in Figure 4. The cracks have been emphasized

\footnotetext{
* Baldwin-Southwork Corp., Philadelphia, $\mathrm{Pa}$.
} 
by painting the surface with a dye etchant.

The stress pattern of the labial and lingual aspect of the maxillary anterior bridge loaded on the left central incisor is illustrated in Figure 5. On the labial aspect the cracks in the lacquer were generally vertical, showing that the strain in tension was in a horizontal direction since the direction of the cracks are at right angles to the strain in tension. The cracks appeared first at the gingival area and proceeded toward the incisal direction. The cracks also generally started at a sharp edge or corner and progressed toward the flat areas. The stress pattern was quite different on the lingual aspect of this bridge and the cracks were more nearly horizontal both on the pontics and the abutments. The tensile strain was, therefore, in the vertical direction. This occurred because not only was the bridge being deflected in a downward direction but it was being tipped forward. As a result, the tensile strain was practically in a vertical direction on the pontic. The restraint of the pin-ledge abutment preparation and the abutment tooth modified the direction of the strain so that it was directed more in a mesial direction. It should be noted that low loads of $0-15$ pounds produced more nearly horizontal cracks than the higher loads of $16-30$ pounds. The stress occurring on the gingival portion of the abutment preparations and the central incisor pontics at a load of $0-15$ pounds was $11,200 \mathrm{lb} / \mathrm{sq}$ inch. Loads of 16-30 pounds were required to produce stresses of $11,200 \mathrm{lb} / \mathrm{sq}$ inch on the more incisal portions of the abutments and on the labial aspect of the left central and lateral incisor pontics.

The corresponding stress patterns on the maxillary anterior bridge with the load applied to the left lateral incisor are shown in Figure 6. In general, higher loads were required to produce cracks in the lacquer, with most of the cracks appearing at loads of $31-60$ pounds. This is understandable considering the load was applied to the lateral incisor which is adjacent to the abutment. Again the cracks were more nearly horizontal on the lingual than on the labial aspect of the bridge, indicating a tipping action. Likewise, the cracks were formed at sharp lines and angles, and, as expected, higher strains occurred in the pontic directly under the load and the adjacent abutment than in the opposite pontic and abutment.

Typical stress patterns for the mandibular anterior bridge with the load applied to the left central incisor are pictured in Figure 7. Considerably less load was required to produce cracks on the lingual than on the labial aspect of the bridge, and the cracks on the former again were more nearly horizontal. The tipping of the bridge in a labial direction may not be typical of conditions in the mouth where a load in a lingual direction might occur. The tipping of the bridge and the reasonably rigid supporting tooth resulted in a tensile strain on the

\section{MAXILLARY ANTERIOR}
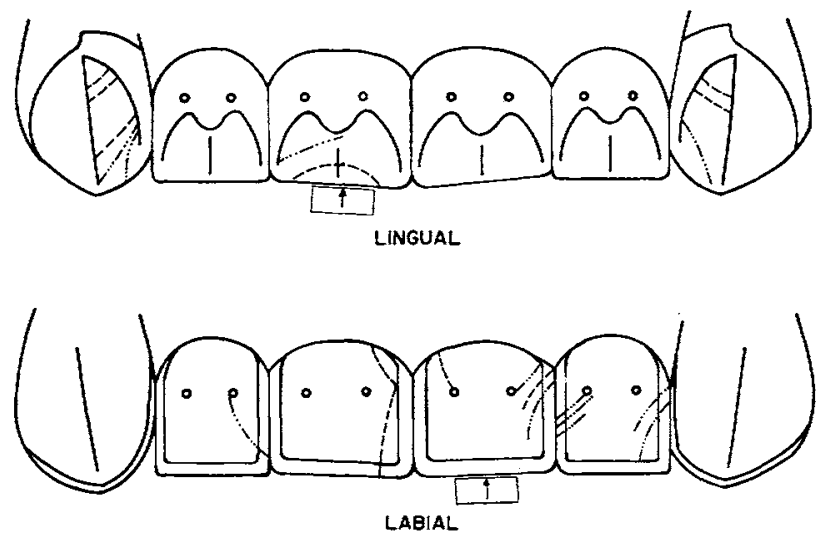

FIG. 5.-Stress pattern observed in the maxillary anterior bridge when the load is applied to the incisa 1

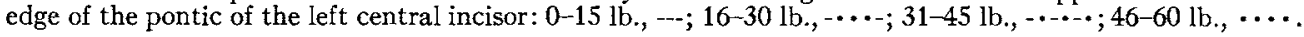



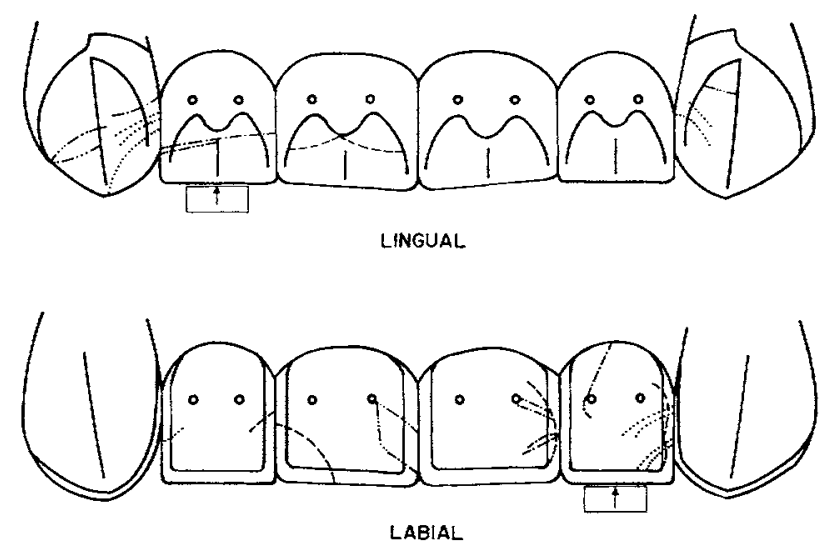

FIG. 6.- Stress pattern in the maxillary anterior bridge with the load applied to the incisal edge of the pontic of the left lateral incisor: $0-15 \mathrm{lb} .,-\cdots ; 16-30 \mathrm{lbs} .,-\cdots, 31-45 \mathrm{lb} ., \cdots \cdots ; 46-60 \mathrm{lb} ., \cdots \cdots$.

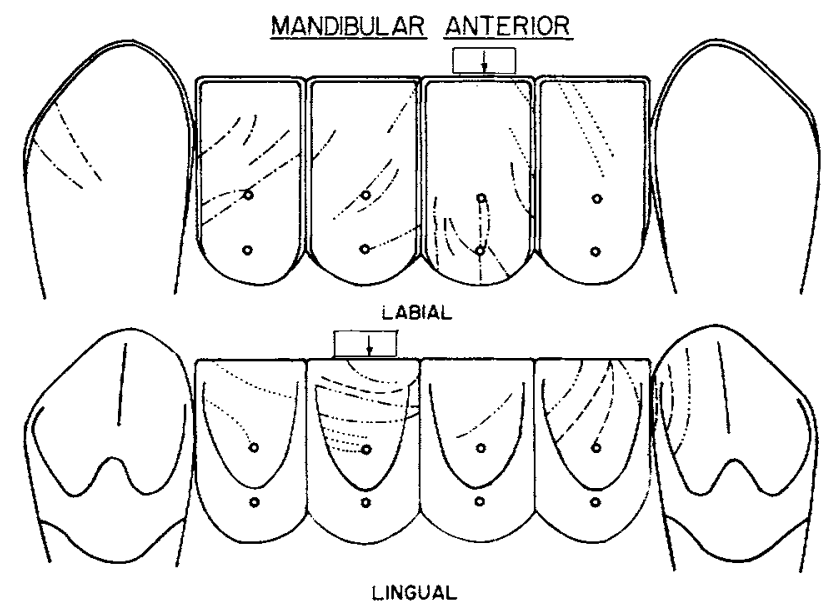

FIG. 7.-Stress pattern in the mandibular anterior bridge with the load applied to the incisal edge of the pontic of the left central incisor: 0-15 lb., --; $16-30 \mathrm{lb} .,-\cdots-; 31-45 \mathrm{lb},, \cdots+\cdots ; 46-60 \mathrm{lb} ., \cdots \cdots$.

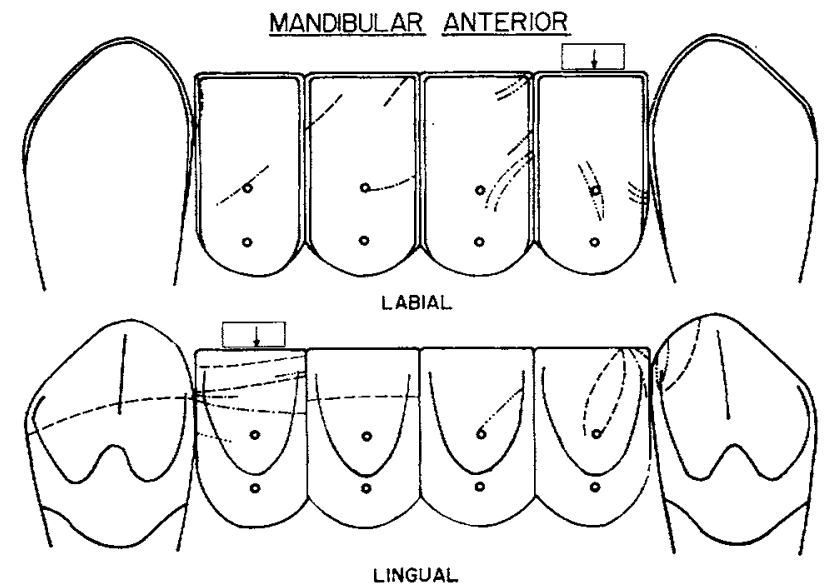

Fig. 8.--Stress pattern in the mandibular anterior bridge with the load applied to the incisal edge of the

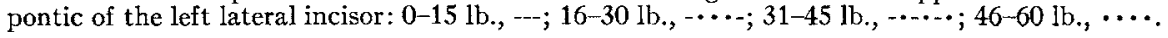


lingual aspect of the right lateral pontic and the $\frac{3}{4}$-crown preparation on the right cuspid in the direction of the load application. Similar cracks did not appear on the left lateral incisor until $46-60$ pounds as a result of differences in the length of the span and the bulk of the preparations. Cracks on the labial aspect of the mandibular bridge were nearly vertical in the gingival area of the left central pontic, showing that the tension strain is in a horizontal direction at this position. The strains in tension in the adjoining pontics are in the direction of the incisal portion of the cuspid abutments from the gingival area of the left central pontic. The $\frac{3}{4}$-crown preparations appeared to function well and remained cemented and the margins closed at the highest load of 60 pounds.

The stress patterns for the mandibular anterior bridge loaded on the left lateral incisor are shown in Figure 8. Similar trends were observed in the strain cracks, but fewer cracks appeared in the gingival areas of the pontics at the higher loads as a result of the support of the adjacent abutment. The cracks again formed at sharp lines and angles and in some instances the holes for retaining the facings. As usual, the lacquer was strained more severely in the area adjacent to the load.

The maxillary posterior bridge was loaded by means of $\frac{1}{8}$-inch diameter ball bearing positioned in the central fossa of the left first molar. The stress pattern is shown in Figure 9, and this bridge is distinguished by the scarcity of cracks in the lacquer even at the highest load of 60 pounds. Cracks did appear at the junctions of the pontics and in the area of the soldered joints between the pontics and the abutment preparation. Cracks appeared in these areas sooner when the bulk of metal at the joint was smaller. One crack was observed on the buccal aspect of the first molar pontic which started at one of the holes for the pin used to retain the facing. Another crack formed on the buccal surface of the full-crown preparation, while the lingual aspect of the bridge was devoid of cracks. Flaking of the lacquer coating was observed at the gingival margin of the full crown, and this indicated compressive stresses in this area. The compressive stress in the lacquer probably resulted from down- ward motion of the molar pontic and the rigid support of the second molar abutment tooth. This force is also responsible for the strain crack on the buccal surface of the full crown which started near the buccal groove.

The maxillary posterior bridge was also loaded on the buccal cusp of the second bicuspid, as shown in Figure 10. Again very few cracks were observed while loading the bridge to 60 pounds. Cracks were formed at the junction of the molar and bicuspid pon-
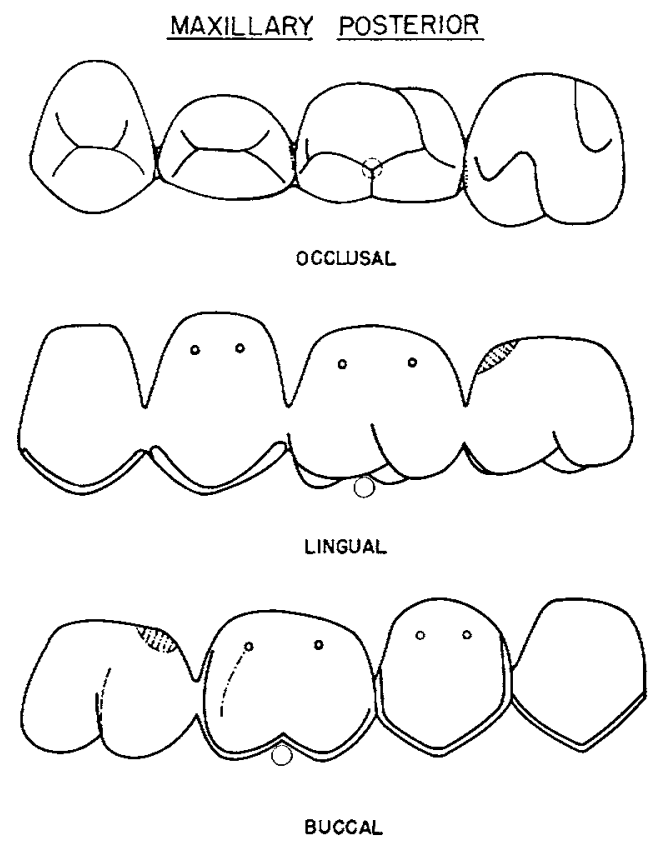

FIG. 9.-Stress pattern in the right maxillary posterior bridge with the load applied through a ball positioned at the central fossa of the pontic of the right first molar: 0-15 lb., ---; 16-30 lb, -...-; 31-45 lb., -.-*; 46-60 lb., $\cdots$.

tics along the sharp line angles, with the direction of the strain in tension being generally vertical. The gingival area of the buccal surface of the full crown was placed in compression as a result of this loading position. Strain cracks were observed in the interproximal spaces, with the first crack forming at $31-45$ pounds between the pontics and at 46-60 pounds between the pontics and the abutment preparations on the lingual aspect. 


\section{Summary}

The magnitude and direction of strains and stresses were determined on fixed-bridge restorations, using a brittle lacquer-coating technique. A maxillary anterior six-unit bridge having pin-ledge preparations on the cuspids, a mandibular anterior six-unit bridge having $\frac{3}{4}$-crowns on the cuspids and a

MAXILLARY POSTERIOR

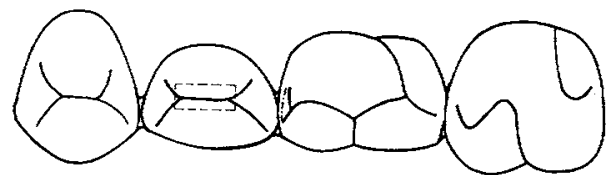

OCCLUSAL

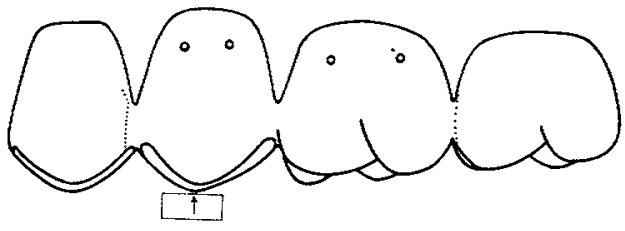

LINGUAL

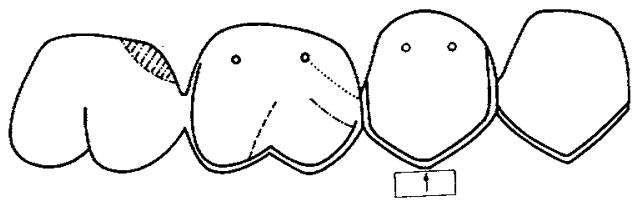

BUCCAL

FIG. 10.- Stress pattern in the right maxillary posterior bridge with the load applied to the buccal cusp of the right second bicuspid: $0-15, \mathrm{lb} .,--; 16-$ 30 lb., -...; 31-45 lb., .....; 46-60 lb., ....

four-unit maxillary bridge having a $\frac{3}{4}$-crown and full crown on the first biscuspid and second molar, respectively, were used in the study.

The maxillary and mandibular anterior bridges were loaded on the incisal edge and were shown to deflect downward and to tip forward during loading. The two anterior bridges, therefore, when loaded sustained tensile strains in a vertical direction on the lingual aspect and in a horizontal direction on the labial aspect. The pin-ledge preparations were not particularly satisfactory, since often the cement failed at the abutments during loading. The $\frac{3}{4}$-crown preparation appeared to be more suitable, since the mandibular bridge having these preparations withstood loading up to 60 pounds. The posterior bridge when loaded deflected in a typical transverse manner, since little if any tipping occurred. The stress and direc. tion of the strain were found to be a function of load, position of the force application, and mass and shape of the restoration.

\section{References}

1. Marler, D. B., and Peyton, F. A. Photoelasticity as a Research Technique for Analyzing Stresses in Dental Structures, $J$. dent. Res., 34:381-88, 1955

2. Noonan, M. A. The Use of Photoelasticity in a Study of Cavity Preparations, J. Dent. Child., 16:24-28, 1949.

3. Hollenger, H. H. Photography in the Photoelastic Stress Analysis of Restorations, Dent. Radiog. Pholog. $31: 31,1958$.

4. Matthews, E., and WaIN, E. A. Stresses in Denture Bases, Brit. dent. J, 100:167-71, 1956.

5. Окомото, K. Distribution of Stresses in the Mandible by Occlusal Movements, $J$. Yonago med. Ass., $9: 791-$ $803,1958$.

6. Miyauchr, T., and Kubota, H. Three Dimensional Photoelasticity as a Method of the Stress Analysis of the Tooth, Shika Gakuho, 57:55-59, 1957.

7. ITo, H. Mechanic of Transposition of the Tooth, $J$. Yonago med. Ass., 10:80-98, 1959.

8. OotawA, H., Effects of the Clasp Rest upon the Tooth. I. Lines of Principal Stresses, Shika Gakuho, 55:329-41, 1955 .

9. Anderson, D. J. Methor of Measuring Masticatory Loads, $J$. dent. Res., 32: 785-89, 1953.

10. Howell, A. H., and Manly, R. S. An Electronic Strain Gauge for Measuring Oral Forces, $J$. dent. Res., $27: 705-12,1948$

11. Howele, A. H., and Brudevold, F. Vertical Forces Used during Chewing of Food, $J$, dent. Res., 29:133 42,1950 .

12. Anderson, D. J. Measurement of Stresses in Mastication. I and II, $J$. dent. Res., 35:664-70, 671-73, 1956.

13. Anderson, D. J., and Picton, D. C. A. Masticatory Stresses in Normal and Modified Occlusion, $J$. dent. Res., $37: 312-17,1958$.

14. Sharry, J. J., Askew, H. C., and Hoyer, H. Influence of Artifical Tooth Forms on Bone Deformation beneath Complete Dentures, $f$. dent. Res., 39:253$66,1960$.

15. Evans, R. G., and Lissner, H. R. "Stresscoat" Deformation Studies of the Femur under Static Vertical Loading, Anat. Res., 100:159, 1948.

16. Gurdian, E. S., and Lissner, H. R. Deformation of the Skull in Head Injury: A Study with "Stresscoat" Technique, Surg. Gynecol. Obstet., $81: 679-82$, 1947.

17. Huflke, D. F. Mechanics in the Production of Mandibular Fracture: A Study with "Stresscoat" Technique. Part I. Symphyseal Impact, $J$. dent. Res., 40:1042-56, 1961.

18. DeForest, A. V., Ellis, G., and STern, F. B., JR. Brittle Coating for Quantitative Strain Measurements, J.appl. Mech., Trans. A.S.M.E., 64A:184-88, 1942 . 